\title{
Initial combination therapy versus step-up therapy in treatment to the target of remission in daily clinical practice in early rheumatoid arthritis patients: results from the DREAM registry
}

L. M. M. Steunebrink ${ }^{1,2^{*}}$, G. A. Versteeg ${ }^{1,2}$, H. E. Vonkeman ${ }^{1,2}$, P. M. ten Klooster ${ }^{2}$, H. H. Kuper ${ }^{1}$, T. R. Zijlstra ${ }^{3}$, P. L. C. M. van Riel ${ }^{4}$ and M. A. F. J. van de Laar $^{1,2}$

\begin{abstract}
Background: Treat to target (T2T) is widely accepted as the standard of care for patients with rheumatoid arthritis (RA) and has been shown to be more effective than traditional routine care. The objective of this study was to compare the effectiveness of two T2T strategies in patients with early RA: a step-up approach starting with methotrexate (MTX) monotherapy (cohort I) versus an initial disease-modifying antirheumatic drug combination approach (cohort II).

Methods: A total of 128 patients from cohort II were case-control-matched with 128 patients from cohort I on gender, age, and baseline disease activity. Twelve-month follow-up data were available for 121 patients in both cohorts. The primary outcome was the proportion of patients having reached at least one 28-joint Disease Activity Score (DAS28) score <2.6 (remission) during 12 months of follow-up. Secondary outcomes were time until remission was achieved and mean DAS28 scores at 6- and 12-month follow-up.

Results: After 12 months of follow-up, remission was reached at least once in $77.3 \%$ of the patients in cohort II versus $71.9 \%$ in cohort I $(P=0.31)$. Median time until first remission was 17 weeks in cohort II versus 27 weeks in cohort I $(P=0.04)$. A significant time by strategy interaction was found in mean DAS28 scores. Post hoc analysis revealed a significant difference in mean DAS28 scores between both cohorts at 6 months $(P=0.04)$, but not at 12 months $(P=0.36)$.
\end{abstract}

Conclusions: The initial combination strategy resulted in a comparable remission rate after 1 year but a significantly shorter time until remission. At 6 months, mean DAS28 scores were lower in patients with initial combination treatment than in those with step-up therapy. At 12 months, no significant differences remained in mean DAS28 scores or the proportion of patients in remission.

Keywords: Treat-to-target strategies, Early rheumatoid arthritis, Remission, DAS28

\footnotetext{
* Correspondence: Imm.steunebrink@mst.nl

${ }^{1}$ Arthritis Center Twente, Department of Rheumatology, Medisch Spectrum

Twente, PO Box 50 000, 7500, KA, Enschede, The Netherlands

${ }^{2}$ Department Psychology, Health \& Technology, University of Twente,

Enschede, The Netherlands

Full list of author information is available at the end of the article
} 


\section{Background}

Treat to target (T2T) is widely accepted as the standard of care for patients with rheumatoid arthritis (RA) and is now recommended in national and international guidelines $[1,2]$. Under the controlled conditions of randomized clinical trials, T2T aimed at remission or low disease activity leads to good clinical outcomes [3-5]. Furthermore, it has been shown that T2T is more effective than traditional routine care, especially when a protocol treatment strategy is used [6]. Recently, it was shown that T2T also leads to more rapid and higher 28-joint Disease Activity Score (DAS28) remission rates and larger improvements in functional ability and patient assessments than usual care in daily clinical practice [7]. Previous clinical trials have additionally compared the effectiveness of different disease-modifying antirheumatic drug (DMARD) strategies, including initial monotherapy, initial combination therapy, or step-up combination therapy with and without the use of corticosteroids or biologics [5, 8-13]. On the basis of these trials, initial combination therapy appears to be more effective than initial monotherapy $[5,12,14]$, while the combination of methotrexate (MTX) monotherapy with high-dose step-down prednisone seems to have equally good results $[11,15]$. However, the optimal treatment protocol is still not clear.

A challenge in the field of RA management remains the implementation of T2T strategies in daily clinical practice. Healthcare professionals may be reluctant to prescribe complex combination therapies, including high-dose corticosteroids, because of concerns about patient compliance and unfavorable side effects [16]. Moreover, time constraints and problems with organizational structures and processes can complicate successful implementation of T2T in daily clinical practice [17]. T2T starting with MTX monotherapy was successfully implemented in the first Dutch Rheumatoid Arthritis Monitoring (DREAM) registry remission induction cohort [18-20]. Despite very promising results, T2T did not have the same impact on all patients [21]. A relevant proportion of patients still did not reach clinical remission within 12 months of treatment [18]. DREAM registry remission induction cohort I was used in the first observational study to describe the results of implementing a T2T strategy in daily clinical practice. Subsequent studies using different T2T strategies are needed to establish which strategy gives the optimal result in daily clinical practice.

Consequently, a second inception cohort was started (cohort II), in which patients were treated with initial combination DMARD therapy consisting of high-dose MTX and hydroxychloroquine (HCQ) with an optional intramuscular triamcinolone acetonide (TCA) injection at the discretion of the rheumatologist. The aim of the present study was to compare the clinical outcomes of both treatment strategies after 1 year of follow-up.

\section{Methods}

\section{Data selection and study design}

In this study, we used data from the ongoing DREAM registry remission induction strategies I and II, two observational, multicenter cohorts established in 2006 and 2012, respectively. In both cohorts, all adults aged $\geq 18$ years with a clinical diagnosis of RA and a disease duration (time from the diagnosis to the start of therapy) $<1$ year were enrolled consecutively immediately after a clinical diagnosis of RA. For this study, data from two hospitals were used: Medisch Spectrum Twente in Enschede and Isala Klinieken in Zwolle, both in the Netherlands. Both treatment strategies were in line with clinical practice and complied with current guidelines for treatment of RA. Exclusion criteria for both cohorts were use of prednisolone $\geq 10 \mathrm{mg} /$ day or previous or current treatment with DMARDs. The medical ethics committees of the Medisch Spectrum Twente, Enschede and Isala, Zwolle hospitals determined, in accordance with Dutch law, that no ethical approval was required, because all data were collected in the course of regular daily clinical practice. Nonetheless, patients were completely informed about the study, and informed consent was obtained from each patient.

At the time of the present analysis, 137 patients had a follow-up of at least 1 year in cohort II. For the aim of this quasi-experimental study, a total of 128 patients from cohort II could be individually matched with 128 patients from cohort I on sex, age ( \pm 5 years) and baseline disease activity ( \pm 0.5 on the DAS28).

\section{Treat-to-target protocol \\ T2T protocol in cohort I}

Patients were evaluated at $0,8,12,20,24,36$, and 52 weeks and every 3 months thereafter. Per-protocol treatment advice was an initial monotherapy of $15 \mathrm{mg} /$ week MTX, with folic acid taken on the second day after MTX. In case of insufficient response (DAS28 $\geq 2.6$ ) at the first subsequent time point, the following per-protocol treatment steps were advised. After 2 months, the MTX dosage was increased to $25 \mathrm{mg} /$ week; after 3 months, sulfasalazine (SSZ) $2000 \mathrm{mg} /$ day was added. After 20 weeks, the SSZ dosage was increased to $3000 \mathrm{mg} /$ day. In accordance with Dutch reimbursement regulations, anti-tumor necrosis factor (anti-TNF)- $\alpha$ treatment was prescribed at week 24 for patients whose DAS28 remained $\geq 3.2$. In these cases, SSZ was replaced with subcutaneous administration of $40 \mathrm{mg}$ of adalimumab every 2 weeks. At week 36, the frequency of adalimumab was increased to $40 \mathrm{mg} /$ week for patients with DAS28 $\geq 2.6$. At week 52 , adalimumab was exchanged for etanercept $50 \mathrm{mg} /$ week for patients with DAS28 $\geq 3.2$. If at any time point the target of DAS $28<2.6$ was met, medication was held constant. In case of sustained remission ( $\geq 6$ months), medication was gradually reduced and eventually discontinued. In case of a disease 
flare (DAS28 $\geq 2.6$ ), the last effective medication or medication dose was restarted and treatment could subsequently be intensified. In individual patients with contraindications to specific medication, deviations from the protocol were allowed. In patients with an allergy to sulfa drugs (sulfonamides), SSZ was replaced by oral HCQ at a dosage of $400 \mathrm{mg} /$ day. Concomitant treatment with nonsteroidal anti-inflammatory drugs, prednisolone at a dosage of $\leq 10 \mathrm{mg} /$ day, and intraarticular corticosteroid injections was allowed at the discretion of the attending rheumatologist. On clinical indication, the attending rheumatologist was free to diverge from the medication schedule at any time. Further details of the study protocol are reported elsewhere [18].

\section{T2T protocol in cohort II}

Patients were evaluated at months $0,2,4$, and 6 and every 3 months thereafter. Treatment advice was an initial combination therapy of MTX $20 \mathrm{mg} /$ week and HCQ $200 \mathrm{mg}$ twice daily. As bridging therapy, an optional intramuscular triamcinolone injection to a maximum dosage of $120 \mathrm{mg}$ could be given. After 1 month, the MTX dosage was increased to $25 \mathrm{mg} /$ week, independent of disease activity. All patients who started MTX also received folic acid on the second day after MTX. After 2 months, in case of persistent disease activity (DAS28 $\geq$ 2.6), the MTX dosage was further increased to $30 \mathrm{mg} / \mathrm{week}$ and an extra optional intramuscular triamcinolone injection could be administered. After 4 months, in case of moderate to high disease activity (DAS28 $\geq 3.2$ ), a TNF inhibitor (adalimumab, etanercept, or infliximab) was added. In case of low disease activity $(2.6<$ DAS2 $8<3.2)$, the attending rheumatologist could choose between SSZ 2000$3000 \mathrm{mg} /$ day or an intramuscular triamcinolone injection.

Similarly to cohort I, disease activity was assessed using the DAS28 at each subsequent visit. If remission (DAS28 < 2.6) had not been reached, treatment was intensified. If the patient was in DAS28 remission, medication was held constant. In case of sustained remission ( $\geq 6$ months), medication was tapered and eventually discontinued according to a predetermined tapering schedule. In case of disease flare (DAS28 $\geq 2.6$ ), the last effective medication or medication dosage was restarted and treatment could subsequently be intensified. On clinical indication, the attending rheumatologist was free to diverge from the medication schedule at any time. For patients with contraindications to specific medications, deviations from the protocol were also allowed.

\section{Assessments}

At each assessment, data were collected on various clinical and patient-reported outcome measures, including measures of disease activity, health-related quality of life, physical function, and laboratory measures. Disease activity was assessed by trained rheumatology nurses using the DAS28, consisting of swollen and tender joint counts in 28 joints, the erythrocyte sedimentation rate (ESR), and a $100-\mathrm{mm}$ visual analogue scale (VAS) on general health $(0=$ "very good" and $100=$ "very bad") [22]. The Health Assessment Questionnaire Disability Index (HAQ-DI) was used to assess physical function [23]. Furthermore, patients rated their pain on a 100 -mm VAS $(0=$ "no pain" and $100=$ "unbearable pain") and completed the 36-item Short Form Health Survey (SF-36) for assessment of their current physical and mental health status [24].

\section{Outcome measures}

The primary outcome was the proportion of patients having reached at least one DAS28 $<2.6$ (DAS28 remission) after 1 year of follow-up. Secondary outcomes were time until remission was achieved and changes in mean DAS28 scores at 6 months and 1 year.

\section{Statistical analysis}

All statistical calculations were performed using the statistical software package IBM SPSS version 20.0 for Windows (IBM, Armonk, NY, USA). Missing values for ESR (1.8\%) and well-being (0.3\%) were imputed on the basis of swollen and tender joint counts and C-reactive protein (CRP) using single imputation with the expectation-maximization method in SPSS. Descriptive statistics for normally distributed variables or categorical variables were reported as frequencies, means, and standard deviations. If variables were not normally distributed, the median with the corresponding interquartile range was reported. To test for any baseline differences, we performed independent $t$ tests for normally distributed variables, Mann-Whitney $U$ tests for nonnormally distributed variables, and $\chi^{2}$ tests for categorical variables. Differences in the proportion of patients achieving remission were tested using $X^{2}$ tests. To compare time until first moment of DAS28 remission, Kaplan-Meier survival analysis was used with log-rank testing for between-group differences. The course of DAS28 over time was compared between groups by specifying a linear mixed model with strategy and time as fixed factors. As the visit schedules were slightly different between both cohorts, only baseline, 6-month, and 12-month data were used for this analysis. An antedependence structure was used as a model for the covariance matrix because it gave the best fit to the data. Post hoc analysis of covariance (ANCOVA) was performed with baseline DAS28 score as a covariate. For all tests, $P<0.05$ was considered to be significant.

\section{Results}

Table 1 summarizes baseline patient characteristics of both cohorts. All patients had active disease with a mean 
Table 1 Baseline characteristics of patients in both cohorts

\begin{tabular}{|c|c|c|c|}
\hline Characteristics & Cohort I $(n=128)$ & Cohort II $(n=128)$ & $P$ value \\
\hline Female sex, $n(\%)$ & $79(61.7 \%)$ & $79(61.7 \%)$ & 1.00 \\
\hline Age, years, mean $\pm S D$ & $59.1 \pm 13.0$ & $59.5 \pm 12.8$ & 0.81 \\
\hline DAS28-ESR, mean \pm SD & $4.5 \pm 1.1$ & $4.8 \pm 1.1$ & 0.02 \\
\hline ESR, mm/h, median (IQR) & $22.0(14.0-41.0)$ & $29.0^{\mathrm{a}}(14.0-45.0)$ & 0.20 \\
\hline CRP, mg/L, median (IQR) & $10.0^{\mathrm{b}}(5.0-22.0)$ & $11.5(4.3-24.8)$ & 0.79 \\
\hline Anti-CCP-positive, $n$ (\%) & $74^{\complement}(58.3 \%)$ & $77^{c}(60.2 \%)$ & 0.93 \\
\hline RF-positive, $n$ (\%) & $62(48.4 \%)$ & $76(59.4 \%)$ & 0.11 \\
\hline $\mathrm{SJC}, n$, median (IQR) & $6.0(3.0-9.0)$ & $5.0(2.0-10.0)$ & 0.24 \\
\hline TJC, $n$, median (IQR) & $3.0(1.0-7.0)$ & $4.0(2.0-10.0)$ & 0.03 \\
\hline HAQ-SDI, median (IQR) & $1.19^{d}(0.88-1.63)$ & $1.0^{\mathrm{e}}(0.38-1.50)$ & $<0.01$ \\
\hline VAS well-being, median (IQR) & $50.0(28.3-65.0)$ & $51.0(35.0-70.0)$ & 0.29 \\
\hline VAS pain, median (IQR) & $50.0^{f}(39.8-64.0)$ & $62.0^{9}(49.0-75.0)$ & $<0.01$ \\
\hline SF-36-PCS, mean $\pm S D$ & $38.1 \pm 7.6^{h}$ & $37.3 \pm 9.2^{i}$ & 0.51 \\
\hline SF-36-MCS, mean \pm SD & $40.7 \pm 7.4^{j}$ & $44.9 \pm 11.9^{k}$ & 0.00 \\
\hline $\mathrm{BMI}, \mathrm{kg} / \mathrm{m}^{2}$, mean $\pm \mathrm{SD}$ & $26.5 \pm 4.8^{\prime}$ & $26.0 \pm 4.1^{\mathrm{m}}$ & 0.39 \\
\hline
\end{tabular}

DAS28-ESR Disease Activity Score in 28 joints using erythrocyte sedimentation rate, CRP C-reactive protein, TJC tender joint count, SJC swollen joint count, HAQ-SDI Health Assessment Questionnaire Disability Index (standard scoring), SF-36 36-item Short Form Health Survey (version 2), PCS Physical Component Summary, MCS Mental Component Summary, BMI body mass index, $R F$ rheumatoid factor, $C C P$ cyclic citrullinated peptide, VAS visual analogue scale $\mathrm{a}_{n=127}$

$\mathrm{b}_{n=121}$

$c_{n=127}$

$\mathrm{d}_{n}=102$

$\mathrm{e}_{n}=87$

$f^{\prime}=122$

$g_{n}=72$

$\mathrm{h}_{n}=105$

in $=86$

$\mathrm{i} n=105$

${ }^{k} n=86$

$' n=126$

$m_{n=119}$

DAS28 of $4.8 \pm 1.1$ in cohort II versus $4.5 \pm 1.1$ in cohort I. Twelve-month data were available for 121 patients $(94.5 \%)$ in both cohorts. In cohort I, seven patients were lost to follow-up, of whom one had received another diagnosis (systemic lupus erythematosus), four were lost to follow-up for unknown reasons, and two had no 12month visit (but were still in the cohort). In cohort II, seven patients were lost to follow-up, of whom one died, one achieved medication-free remission, one was misdiagnosed as having arthritis, two were lost to follow-up for unknown reasons, and two had no 12-month visit (but were still in the cohort).

\section{Remission}

First remission within 6 months was achieved in $63.3 \%$ (81 of 128) of the patients in cohort II versus $48.4 \%$ (62 of 128) in cohort I $(P=0.02)$. First remission within 12 months was achieved in $77.3 \%(n=99$ of 128$)$ of the patients in cohort II versus $71.9 \%$ (92 of 128$)$ in cohort I $(P=0.31)$. Median time until first remission was 17 weeks $(95 \%$ confidence interval $[\mathrm{CI}] 13.2-20.8)$ in cohort II versus 27 weeks $(95 \%$ CI 20.7-33.3) in cohort I $(P=0.04)$ (Fig. 1$)$.
The proportion of patients in remission in cohorts II and $\mathrm{I}$ at the time of the 6-month assessments were $61.7 \%$ (74 of 120$)$ and $45.9 \%$ (56 of 122$)(P=0.01)$, respectively. The proportion of patients in remission in cohorts II and I at the 12-month assessments were $63.6 \%$ (77 of 121$)$ and $60.3 \%(n=73$ of 121$)(P=0.60)$. The majority of the patients in both cohorts reached their first remission using the initial medication only. In cohort II, $84.8 \%$ achieved remission with MTX + HCQ, while in cohort I $63.0 \%$ achieved remission with MTX monotherapy.

\section{Course over time of the DAS28}

Figure 2 represents the mean DAS28 over time in both cohorts. A significant time by strategy interaction was found $(P=0.01)$, indicating a different pattern of DAS28 change over time between both cohorts. At 6 months, the mean DAS28 in cohort II was below the cutoff for remission (DAS28 $<2.6$ ), while the mean DAS28 in cohort I was still above remission (DAS28 > 2.6). At 12 months, mean DAS28 was $<2.6$ in both cohorts. According to the post hoc ANCOVA, mean DAS28 scores 


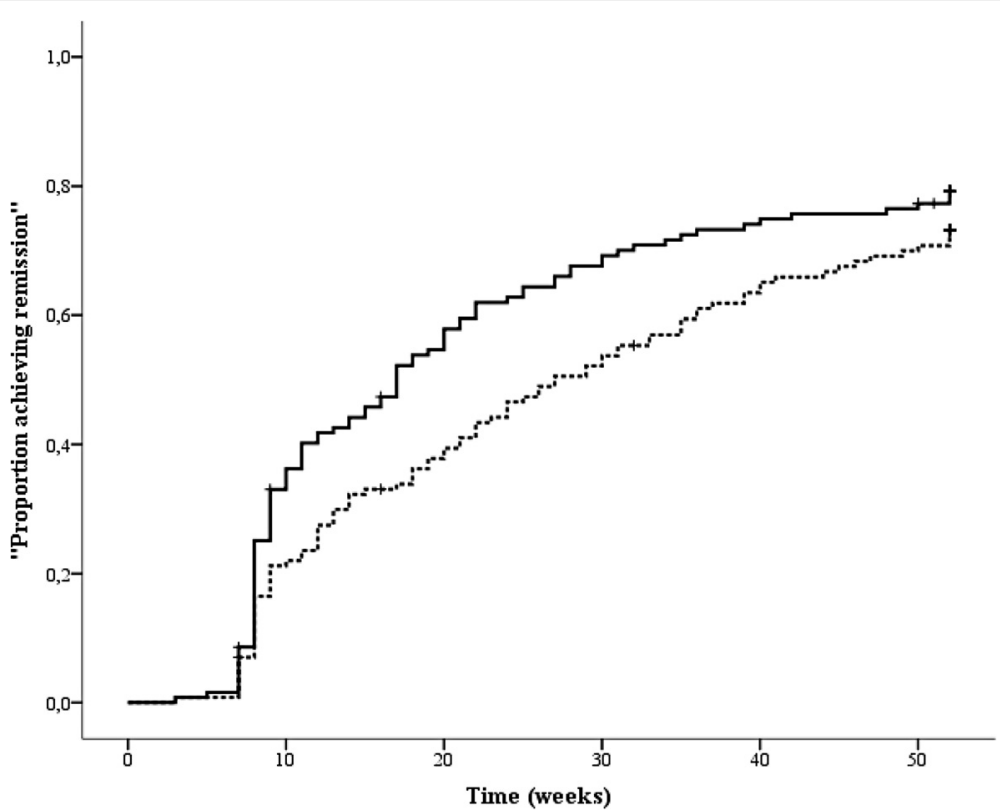

Fig. 1 Kaplan-Meier curves for time until first remission was reached. The solid line represents cohort II, and the dashed line represents cohort

were significantly lower in cohort II at 6 months (mean difference $0.30,95 \%$ CI $0.01-0.58, P=0.04)$ but not at 12 months (mean difference $0.13,95 \% \mathrm{CI}-0.14$ to 0.40 , $P=0.36)$.

\section{Medication use}

At baseline, 67 patients (53.3\%) in cohort II received an intramuscular injection with triamcinolone versus 4 patients $(3.1 \%)$ in cohort I $(P<0.01)$. Receiving an injection with triamcinolone in cohort II was associated with a higher odds ratio (OR 2.14, $95 \%$ CI 1.03-4.46, $P=$ 0.04) of achieving first remission within 6 months but no longer with achieving remission within 12 months (OR 1.77, $95 \%$ CI 0.77-4.10, $P=0.018$ ). The actual medication used at 12 months (Table 2) was significantly different between both cohorts $(P=0.03)$. The vast

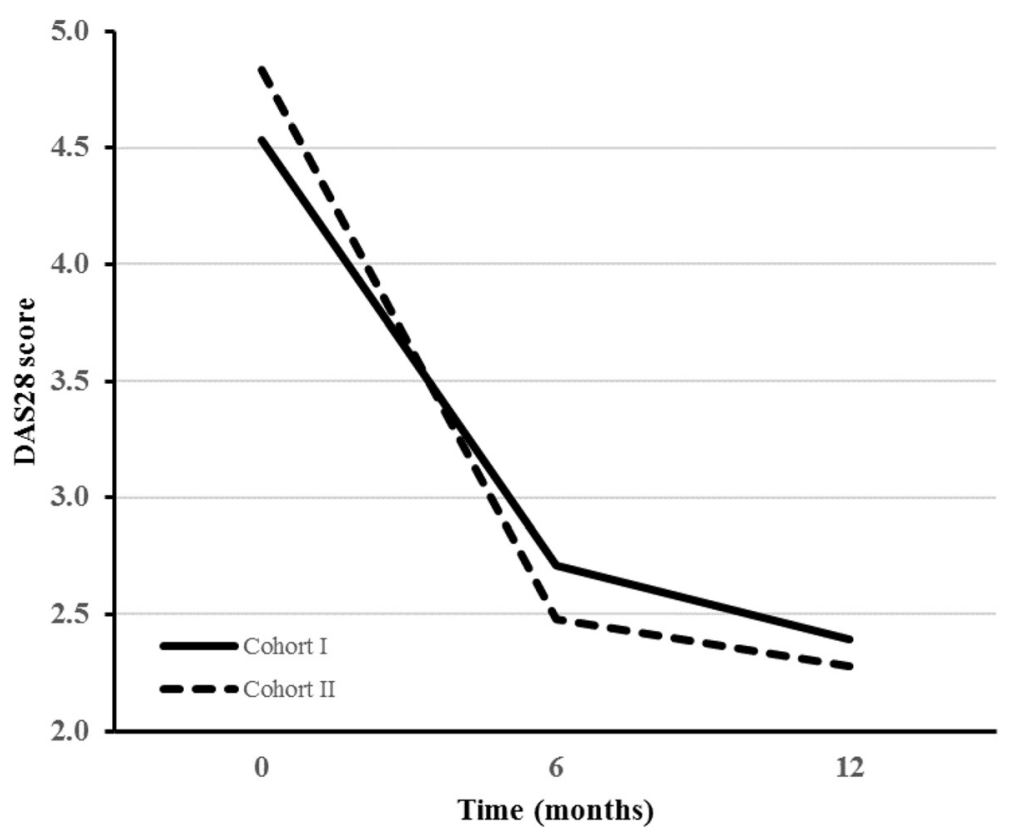

Fig. 2 Decrease in mean 28-joint Disease Activity Score (DAS28) in treat to target cohort II versus cohort I in 1 year. The dashed line represents cohort II, and the solid line represents cohort I 
Table 2 Medication at 12 months

\begin{tabular}{lll}
\hline Medication & Cohort I $(n=128)$ & Cohort II $(n=128)$ \\
\hline No DMARD & $2(1.6 \%)$ & $10(7.8 \%)$ \\
csDMARD only & $116(90.6 \%)$ & $104(81.3 \%)$ \\
bDMARD & $5(3.9 \%)$ & $11(8.6 \%)$ \\
Lost to follow-up & $5(3.9 \%)$ & $3(2.3 \%)$ \\
\hline
\end{tabular}

$D M A R D$ disease-modifying antirheumatic drug, cSDMARD conventional synthetic disease-modifying antirheumatic drug, $C D M A R D$ biologic disease-modifying antirheumatic drug

majority of the patients in both cohorts received conventional synthetic disease-modifying antirheumatic drugs (csDMARDs) only at 12 months. A small percentage of the patients in both cohorts were prescribed a biologic DMARD. In cohort II, there were slightly more patients for whom DMARD therapy was fully discontinued.

\section{Discussion}

The results of this study suggest that the previously reported outcomes that were achieved by implementation of T2T with initial MTX monotherapy in daily clinical practice may be reproduced, and even improved upon, using an initial combination approach. In the present study, DAS28 remission was reached at least once in $77.3 \%$ of the patients in cohort II versus $71.9 \%$ in cohort I in 1 year of follow-up. Although after 1 year there was no significant difference in the number of patients who had reached remission, remission was reached significantly more rapidly, with a median time to first remission of 17 weeks in cohort II versus 27 weeks in cohort I. A significant difference between strategies in mean DAS28 scores was also found at 6 months, but no longer at 12 months. Therefore, T2T using initial combination therapy may not be superior in the number of patients achieving remission in the longer term, but the strategy does lead to a significantly shorter time until first remission is achieved.

Researchers in various T2T studies in patients with recent-onset RA have reported remission rates varying from $10 \%$ to $78 \%$ [25]. However, it should be noted that these remission rates are difficult to compare, as the investigators in these studies used different criteria for remission and reported slightly different follow-up periods. The TICORA study had the highest proportion $(65 \%)$ of patients in remission (defined as DAS44<1.6) after 18 months of follow-up so far [4]. In the BeST study, the overall proportion of patients in DAS44 remission at 1 year was $32 \%$, with no differences between the four therapeutic groups [26]. The CAMERA group reported that $35 \%$ of the patients in the intensive group attained remission (defined as no swollen joints and at least two of three of the following criteria: number of tender joints $\leq 3$, ESR $\leq 20 \mathrm{~mm} / \mathrm{h}$, and VAS general wellbeing $\leq 20 \mathrm{~mm}$ ) for a period of 3 months after 1 year of follow-up [3]. The IMPROVED study showed an early DAS44 remission rate of $61 \%$ [15]. The CareRA trial researchers, comparing three different intensive combination strategies with glucocorticoids, reported DAS28 (based on CRP) remission rates ranging from $68.1 \%$ to $73.6 \%$ within 16 weeks [27]. The tREACH trial investigators compared two triple-DMARD induction therapies with MTX monotherapy and reported that approximately $78 \%$ of the patients using triple-DMARD therapy had a DAS $<2.4$ after 3 months, compared with $60 \%$ of the patients using MTX monotherapy [28].

The present study shows that both DREAM registry remission induction cohorts I and II resulted in comparably high proportions of patients achieving remission within 12 months. While the implementation of a protocol T2T may in itself explain the high remission rates in both cohorts, apparently even better results may be attained regarding the time needed to achieve first remission by implementing initial combination protocols in daily clinical practice. This is in accordance with previous randomized clinical trials. Among the different therapeutic strategies, early combination therapy that included high-dose step-down DMARDs and low-dose steroids was shown to be most effective in the management of early RA [8, 26, 29-31]. Low-dose prednisone added to conventional DMARD treatment in patients with early RA induced earlier and better disease activity control and provided greater probability for a more stable clinical remission over time [32]. A strict protocol, frequent monitoring, and the commitment and adherence of doctors and nurses may also contribute to optimal outcomes in daily clinical practice $[6,33]$.

Differences in disease activity and in time until remission are clinically important and are likely to also have significant social and economic impact. For instance, patients with persistently active disease are more likely to discontinue work [34]. The DREAM registry remission induction cohort I already showed the feasibility of sustained remission in daily clinical practice [16]. Cohort II also demonstrated impressive improvements in disease activity, comparable with or even better than that reported in previous studies of combination therapy for RA $[5,8,11-13,18]$. In the present study, mean DAS28 scores were significantly lower in cohort II than in cohort I at 6 months but not at 12 months. This is in line with clinical trials showing that initial combination therapy appears to result in more rapid improvements in disease activity, daily functioning, and quality of life than initial monotherapy [4, 11, 26, 35].

Optimal use of MTX in combination with other DMARDs early in the course of the disease leads to considerable improvement in disease activity [3, 4, 19]. Unfortunately, previous studies show some conflicting results regarding the efficacy of monotherapy versus 
combination therapy. Double- or triple-combination therapy appears to lead to better clinical outcomes than MTX monotherapy [13, 26, 35]. The TEAR trial concluded that combination treatment was more effective than MTX monotherapy before step-up therapy [14]. The CARDERA study, which was not an initial combination therapy, showed that intensive combination therapy with MTX, ciclosporin, and glucocorticosteroids after failure of MTX monotherapy leads to sustained benefits in damage and disability outcomes [36]. However, a recent study done in Belgium (the CareRA trial) showed that in high-risk patients with RA, MTX in combination with a moderate step-down dose of glucocorticosteroids was as effective in inducing remission as DMARD combination therapies with moderate or high step-down glucocorticosteroid doses [27]. In contrast, the tREACH trial showed that a DMARD combination was better than MTX monotherapy, both in combination with low-dose glucocorticoid bridging [12]. In the United States, MTX + HCQ is by far the DMARD combination most commonly prescribed by rheumatologists [37]. It has been shown to be more potent than MTX used alone [38]. An explanation for this may be that HCQ increases the bioavailability of MTX and/or reduces the clearance of the drug [39].

In the present study, the vast majority of the patients in both cohorts achieved the treatment target using csDMARDs only, and a small number of the patients in both cohorts were using biologic DMARDs at 12 months. This highlights again the importance of the initial treatment choice for the majority of the patients.

It should be noted that the present study was a quasiexperimental study of two cohorts separated over time. The first cohort started in 2006 with MTX monotherapy, and the second cohort started in 2012 with a DMARD combination therapy. Although we did not conduct a randomized trial, we still think that the design and results of the study allow us to compare the two cohorts. The cohorts consist of very similar populations of all consecutive newly diagnosed patients with RA treated in the same hospitals by the same rheumatologists. Patients in cohort II were additionally case-controlmatched with patients in cohort I on gender, age, and baseline disease activity. Although baseline disease activity, disability, and pain were slightly but significantly worse in cohort II, the number of patients achieving remission within 6 months was even higher in this cohort. Moreover, post hoc analyses of mean DAS28 scores controlling for baseline disease activity produced very similar results.

Because in our study we used real-life observational data, the results are generalizable to daily clinical practice. Longer follow-up is needed to investigate the sustainability of remission in cohort II. However, previous studies have shown that a shorter time until remission is related to the sustainability of remission, supporting the importance of early intervention with effective therapy to achieve early remission [40]. Although the remission rates in this study were very high, there is still a small percentage of patients who do not achieve remission, thus creating a need to further identify predictors of remission [21].

\section{Conclusions}

Initial RA combination therapy with high-dose MTX, $\mathrm{HCQ}$, and optional intramuscular TCA was not superior in achieving the number of patients in remission after 1 year, but it did result in a shorter time until first remission versus a step-up approach using MTX and SSZ in the setting of T2T therapy in daily clinical practice.

\section{Abbreviations \\ ANCOVA: analysis of covariance; Anti-CCP: anti- cyclic citrullinated peptide; bDMARD: biologic disease-modifying antirheumatic drug; BMl: body mass index; Cl: confidence interval; CRP: C-reactive protein; CSDMARD: conventional synthetic disease-modifying antirheumatic drug; DAS28: 28-joint Disease Activity Score; DMARD: disease-modifying antirheumatic drug; DREAM: Dutch Rheumatoid Arthritis Monitoring registry; ESR: erythrocyte sedimentation rate; HAQ-DI: Health Assessment Questionnaire Disability Index; HCQ: hydroxychloroquine; \\ IQR: interquartile range; MCS: Mental Component Summary; MTX: methotrexate; OR: odds ratio; PCS: Physical Component Summary; RA: rheumatoid arthritis; RF: rheumatoid factor; SD: standard deviation; SF-36: 36-item Short Form Health Survey; SJC: swollen joint count; SSZ: sulfasalazine; T2T: treat to target; \\ TCA: triamcinolone acetonide; TJC: tender joint count; TNF: tumor necrosis factor; VAS: visual analogue scale.}

\section{Competing interests}

The authors declare that they have no competing interests.

\section{Authors' contributions}

All authors participated in the preparation of the manuscript in a significant way. LMMS drafted the first version of the manuscript. PMtK, HEV, GAV, HHK, TRZ, PLCMvR, and MAFJVdL revised the manuscript critically for important intellectual content. LMMS performed the statistical analysis. All authors participated in the interpretation of the results, and all authors read and approved the final manuscript.

\section{Acknowledgments}

The authors thank all the rheumatologists, nurses, and patients who participated in this study.

\section{Author details}

${ }^{1}$ Arthritis Center Twente, Department of Rheumatology, Medisch Spectrum Twente, PO Box 50 000, 7500, KA, Enschede, The Netherlands. ²Department Psychology, Health \& Technology, University of Twente, Enschede, The Netherlands. ${ }^{3}$ Department of Rheumatology, Isala Klinieken, Zwolle, The Netherlands. ${ }^{4}$ Department of Rheumatology, Bernhoven, Uden, The Netherlands.

Received: 27 November 2015 Accepted: 25 February 2016 Published online: 08 March 2016

References

1. Smolen JS, Landewé R, Breedveld FC, Buch M, Burmester G, Dougados M, et al. EULAR recommendations for the management of rheumatoid arthritis with synthetic and biological disease-modifying antirheumatic drugs: 2013 update. Ann Rheum Dis. 2014;73:492-509.

2. Smolen JS, Aletaha D, Bijlsma JWJ, Breedveld FC, Boumpas D, Burmester G, et al. Treating rheumatoid arthritis to target: recommendations of an international task force. Ann Rheum Dis. 2010;69:631-7. 
3. Verstappen SMM, Jacobs JWG, van der Veen MJ, Heurkens AHM, Schenk Y, ter Borg EJ, et al. Intensive treatment with methotrexate in early rheumatoid arthritis: aiming for remission. Computer Assisted Management in Early Rheumatoid Arthritis (CAMERA, an open-label strategy trial). Ann Rheum Dis. 2007;66:1443-9

4. Grigor C, Capell H, Stirling A, McMahon AD, Lock P, Vallance R, et al. Effect of a treatment strategy of tight control for rheumatoid arthritis (the TICORA study): a single-blind randomised controlled trial. Lancet. 2004;364:263-9.

5. Goekoop-Ruiterman YPM, de Vries-Bouwstra JK, Allaart CF, van Zeben D, Kerstens PJSM, Hazes JMW, et al. Clinical and radiographic outcomes of four different treatment strategies in patients with early rheumatoid arthritis (the BeSt study): a randomized, controlled trial. Arthritis Rheum. 2005;52:3381-90.

6. Schipper LG, van Hulst LTC, Grol R, van Riel PLCM, Hulscher MEJL, Fransen J. Meta-analysis of tight control strategies in rheumatoid arthritis: protocolized treatment has additional value with respect to the clinical outcome. Rheumatology (Oxford). 2010;49:2154-64.

7. Schipper LG, Vermeer M, Kuper HH, Hoekstra MO, Haagsma CJ, Den Broeder $\mathrm{AA}$, et al. A tight control treatment strategy aiming for remission in early rheumatoid arthritis is more effective than usual care treatment in daily clinical practice: a study of two cohorts in the Dutch Rheumatoid Arthritis Monitoring registry. Ann Rheum Dis. 2012;71:845-50.

8. Boers M, Verhoeven AC, Markusse HM, van de Laar MA, Westhovens $\mathrm{R}$, van Denderen JC, et al. Randomised comparison of combined step-down prednisolone, methotrexate and sulphasalazine with sulphasalazine alone in early rheumatoid arthritis. Lancet. 1997;350:309-18.

9. Mäkinen $H$, Kautiainen $H$, Hannonen $P$, Möttönen $T$, Leirisalo-Repo $M$, Laasonen $L$, et al. Sustained remission and reduced radiographic progression with combination disease modifying antirheumatic drugs in early rheumatoid arthritis. J Rheumatol. 2007;34:316-21.

10. Heimans L, Wevers-de Boer KVC, Visser K, Goekoop RJ, van Oosterhout M, Harbers JB, et al. A two-step treatment strategy trial in patients with early arthritis aimed at achieving remission: the IMPROVED study. Ann Rheum Dis. 2014;73:1356-61

11. Den Uyl D, ter Wee M, Boers M, Kerstens P, Voskuyl A, Nurmohamed M, et al. A non-inferiority trial of an attenuated combination strategy ('COBRA-light') compared to the original COBRA strategy: clinical results after 26 weeks. Ann Rheum Dis. 2014;73:1071-8.

12. De Jong PH, Hazes JM, Han HK, Huisman M, van Zeben D, van der Lubbe PA, et al. Randomised comparison of initial triple DMARD therapy with methotrexate monotherapy in combination with low-dose glucocorticoid bridging therapy; 1-year data of the tREACH trial. Ann Rheum Dis. 2014;73:1331-9.

13. O'Dell JR, Leff R, Paulsen G, Haire C, Mallek J, Eckhoff PJ, et al. Treatment of rheumatoid arthritis with methotrexate and hydroxychloroquine, methotrexate and sulfasalazine, or a combination of the three medications: results of a two-year, randomized, double-blind, placebo-controlled trial. Arthritis Rheum. 2002;46:1164-70.

14. Moreland LW, O'Dell JR, Paulus HE, Curtis JR, Bathon JM, St Clair EW, et al. A randomized comparative effectiveness study of oral triple therapy versus etanercept plus methotrexate in early aggressive rheumatoid arthritis: the treatment of Early Aggressive Rheumatoid Arthritis Trial. Arthritis Rheum. 2012;64:2824-35.

15. Wevers-de Boer K, Visser K, Heimans L, Ronday HK, Molenaar E, Groenendael $J H L M$, et al. Remission induction therapy with methotrexate and prednisone in patients with early rheumatoid and undifferentiated arthritis (the IMPROVED study). Ann Rheum Dis. 2012;71:1472-7.

16. Van Tuyl LHD, Plass AMC, Lems WF, Voskuyl AE, Dijkmans BAC, Boers M. Why are Dutch rheumatologists reluctant to use the COBRA treatment strategy in early rheumatoid arthritis? Ann Rheum Dis. 2007;66:974-6.

17. Meyfroidt S, van Hulst L, De Cock D, Van der Elst $K$, Joly J, Westhovens R, et al. Factors influencing the prescription of intensive combination treatment strategies for early rheumatoid arthritis. Scand J Rheumatol. 2014;43:265-72.

18. Vermeer M, Kuper HH, Hoekstra M, Haagsma CJ, Posthumus MD, Brus HLM, et al. Implementation of a treat-to-target strategy in very early rheumatoid arthritis: results of the Dutch Rheumatoid Arthritis Monitoring remission induction cohort study. Arthritis Rheum. 2011;63:2865-72.

19. Vermeer M, Kuper HH, Moens HJB, Drossaers-Bakker KW, van der Bijl AE, van Riel PLCM, et al. Sustained beneficial effects of a protocolized treat-to-target strategy in very early rheumatoid arthritis: three-year results of the Dutch Rheumatoid Arthritis Monitoring remission induction cohort. Arthritis Care Res (Hoboken). 2013;65:1219-26.
20. Vermeer M, Kievit W, Kuper HH, Braakman-Jansen LM, Bernelot Moens HJ, Zijlstra TR, et al. Treating to the target of remission in early rheumatoid arthritis is cost-effective: results of the DREAM registry. BMC Musculoskelet Disord. 2013:14:350.

21. Siemons L, Ten Klooster PM, Vonkeman HE, Glas CA, van de Laar MAFJ. Distinct trajectories of disease activity over the first year in early rheumatoid arthritis patients following a treat-to-target strategy. Arthritis Care Res (Hoboken). 2013;66(4):625-30.

22. Prevoo ML, van't Hof MA, Kuper HH, van Leeuwen MA, van de Putte LB, van Riel PL. Modified disease activity scores that include twenty-eight-joint counts. Development and validation in a prospective longitudinal study of patients with rheumatoid arthritis. Arthritis Rheum. 1995;38:44-8.

23. Bruce B, Fries JF. The Health Assessment Questionnaire (HAQ). Clin Exp Rheumatol. 2005;23(5 Suppl 39):S14-8.

24. Ware JE, Sherbourne CD. The MOS 36-item short-form health survey (SF-36). I. Conceptual framework and item selection. Med Care. 1992;30:473-83.

25. Van der Kooij SM, Allaart CF, Dijkmans BA, Breedveld FC. Innovative treatment strategies for patients with rheumatoid arthritis. Curr Opin Rheumatol. 2008;20:287-94.

26. Goekoop-Ruiterman YPM, de Vries-Bouwstra JK, Allaart CF, van Zeben D, Kerstens PJSM, Hazes JMW, et al. Clinical and radiographic outcomes of four different treatment strategies in patients with early rheumatoid arthritis (the BeSt study): A randomized, controlled trial. Arthritis Rheum. 2008;58(2 Suppl):S126-35.

27. Verschueren P, De Cock D, Corluy L, Joos R, Langenaken C, Taelman V, et al. Methotrexate in combination with other DMARDs is not superior to methotrexate alone for remission induction with moderate-to-high-dose glucocorticoid bridging in early rheumatoid arthritis after 16 weeks of treatment: the CareRA trial. Ann Rheum Dis. 2015;74:27-34.

28. De Jong PH, Hazes JM, Barendregt PJ, Huisman M, van Zeben D, van der Lubbe PA, et al. Induction therapy with a combination of DMARDs is better than methotrexate monotherapy: first results of the tREACH trial. Ann Rheum Dis. 2013;72:72-8.

29. Wassenberg S, Rau R, Steinfeld P, Zeidler H. Very low-dose prednisolone in early rheumatoid arthritis retards radiographic progression over two years: a multicenter, double-blind, placebo-controlled trial. Arthritis Rheum. 2005;52:3371-80.

30. Kirwan JR. The effect of glucocorticoids on joint destruction in rheumatoid arthritis. The Arthritis and Rheumatism Council Low-Dose Glucocorticoid Study Group. N Engl J Med. 1995;333:142-6.

31. Svensson B, Boonen A, Albertsson K, van der Heijde D, Keller C, Hafström I. Low-dose prednisolone in addition to the initial disease-modifying antirheumatic drug in patients with early active rheumatoid arthritis reduces joint destruction and increases the remission rate: a two-year randomized trial. Arthritis Rheum. 2005:52:3360-70.

32. Todoerti M, Scirè CA, Boffini N, Bugatti S, Montecucco C, Caporali R. Early disease control by low-dose prednisone comedication may affect the quality of remission in patients with early rheumatoid arthritis. Ann N Y Acad Sci. 2010;1193:139-45.

33. Vermeer M, Kuper HH, Bernelot Moens HJ, Hoekstra M, Posthumus MD, van Riel PL, et al. Adherence to a treat-to-target strategy in early rheumatoid arthritis: results of the DREAM remission induction cohort. Arthritis Res Ther. 2012;14:R254.

34. Puolakka K, Kautiainen H, Möttönen T, Hannonen P, Korpela M, Julkunen H, et al. Impact of initial aggressive drug treatment with a combination of disease-modifying antirheumatic drugs on the development of work disability in early rheumatoid arthritis: a five-year randomized followup trial. Arthritis Rheum. 2004;50:55-62.

35. Möttönen $T$, Hannonen $P$, Leirisalo-Repo M, Nissilä M, Kautiainen $H$, Korpela $M$, et al. Comparison of combination therapy with single-drug therapy in early rheumatoid arthritis: a randomised trial. FIN-RACo trial group. Lancet. 1999:353:1568-73.

36. Choy EHS, Smith CM, Farewell V, Walker D, Hassell A, Chau L, et al. Factorial randomised controlled trial of glucocorticoids and combination disease modifying drugs in early rheumatoid arthritis. Ann Rheum Dis. 2008:67:656-63.

37. Mikuls TR, O'Dell J. The changing face of rheumatoid arthritis therapy: results of serial surveys. Arthritis Rheum. 2000:43:464-5.

38. Trnavský K, Gatterová J, Lindusková M, Pelisková Z. Combination therapy with hydroxychloroquine and methotrexate in rheumatoid arthritis. Z Rheumatol. 1993;52:292-6. 
39. Carmichael SJ, Beal J, Day RO, Tett SE. Combination therapy with methotrexate and hydroxychloroquine for rheumatoid arthritis increases exposure to methotrexate. J Rheumatol. 2002;29:2077-83.

40. Schipper LG, Fransen J, den Broeder AA, Van Riel PLCM. Time to achieve remission determines time to be in remission. Arthritis Res Ther. 2010;12:R97.

Submit your next manuscript to BioMed Central and we will help you at every step:

- We accept pre-submission inquiries

- Our selector tool helps you to find the most relevant journal

- We provide round the clock customer support

- Convenient online submission

- Thorough peer review

- Inclusion in PubMed and all major indexing services

- Maximum visibility for your research

Submit your manuscript at www.biomedcentral.com/submit 